\title{
A New Definition of A Priori Knowledge: In Search of a Modal Basis
}

\section{Tahko, Tuomas}

2008

Tahko, T 2008 , ' A New Definition of A Priori Knowledge: In Search of a Modal Basis ', Metaphysica, vol. 9 , no. 1 , pp. 57-68 . https://doi.org/10.1007/s12133-007-0022-7

http://hdl.handle.net/10138/27788

https://doi.org/10.1007/s12133-007-0022-7

publishedVersion

Downloaded from Helda, University of Helsinki institutional repository.

This is an electronic reprint of the original article.

This reprint may differ from the original in pagination and typographic detail.

Please cite the original version. 


\title{
A New Definition of A Priori Knowledge: In Search of a Modal Basis
}

\author{
Tuomas E. Tahko
}

Published online: 9 October 2007

(C) Springer Science + Business Media B.V. 2007

\begin{abstract}
In this paper I will offer a novel understanding of a priori knowledge. My claim is that the sharp distinction that is usually made between a priori and a posteriori knowledge is groundless. It will be argued that a plausible understanding of a priori and a posteriori knowledge has to acknowledge that they are in a constant bootstrapping relationship. It is also crucial that we distinguish between a priori propositions that hold in the actual world and merely possible, non-actual a priori propositions, as we will see when considering cases like Euclidean geometry. Furthermore, contrary to what Kripke seems to suggest, a priori knowledge is intimately connected with metaphysical modality, indeed, grounded in it. The task of a priori reasoning, according to this account, is to delimit the space of metaphysically possible worlds in order for us to be able to determine what is actual.
\end{abstract}

Keywords A priori $\cdot$ A posteriori $\cdot$ Knowledge $\cdot$ Necessity $\cdot$ Possibility

\section{Introduction}

Characterisations of the a priori usually start with the idea that a proposition is knowable a priori if it is knowable independently of experience. But to what degree can anything be known independently of experience? What is the relationship between established a posteriori knowledge and a priori knowledge? And further, are there any synthetic a priori truths, or, more neutrally, non-analytic a priori truths? I suppose that the most popular view today is that all a priori truths are analytic and that a priori reasoning is some sort of conceptual analysis. However, the alternative view, namely that some and indeed the most interesting kind of a priori truths are not analytic, is not unheard of. It does certainly seem that if there are non-analytic a priori truths, they are the most interesting sort. Here, I am interested in the a priori

T. E. Tahko $(\bowtie)$

Department of Philosophy, Durham University, 50 Old Elvet, Durham DH1 3HN, UK

e-mail: tuomas.tahko@durham.ac.uk 
exactly in this sense. I will suggest that the (non-analytic) a priori, rather than strictly independent of experience, is always one step beyond experience. To be able to reach this step, we must have an a posteriori framework to take us just below that next step. Furthermore, once the a priori step has been firmly verified, by a posteriori methods, it becomes a part of the established a posteriori framework. What I mean is that the a priori is in a constant bootstrapping relationship with the a posteriori. Despite this intimate connection with the a posteriori, a priori reasoning is a distinct, crucial method of inquiry which is not reducible to the empirical. In Section 2, I will examine the connection between the a posteriori and the a priori and defend the view that they are in a bootstrapping relationship.

Apart from the analytic/synthetic distinction, it needs to be settled where the a priori stands in terms of the necessary/contingent distinction. I will argue that the defining characteristic of the a priori is in fact its relationship with modality. To start with, we must acknowledge Kripke's critique: 'a priori' is not synonymous with 'necessary', and not all necessary truths are a priori. Given Kripke's compelling examples, there should be very little controversy over this matter. Nevertheless, I certainly wish to maintain the link between the a priori and modality; the qualification that is needed concerns the strength of this connection, namely, apriority only implies possibility. In what follows, it will be shown that it is precisely the connection with modality that helps us to answer some of the hardest questions about the a priori. For example, the question about the status of the a priori in regard to the analytic/synthetic distinction then reduces into a question about the nature of the involved modality. If the modality in question is conceptual, it would appear that there is little room for non-analytic a priori truths, but if a priori knowledge concerns metaphysical modality, it seems clear that there have to be (something like) synthetic a priori truths. I will argue that the latter is true, although I do not find the analytic/synthetic distinction very informative in the first place. The relationship between the a priori and modality is the concern of Section 3. Finally, I will combine the two points introduced above - the bootstrapping relationship between a posteriori and a priori knowledge and the connection between the a priori and metaphysical modality - and demonstrate that the upshot of these views is a coherent and plausible characterisation of the a priori.

Before I will advance to defend my claims in detail, it will be necessary to make some clarifications. Firstly, the positive characterisation of 'a priori knowledge' suggested here states that a logically valid a priori proposition ${ }^{1}$ always holds in at least one possible world. It is a separate issue whether it holds in the actual world, and this generally has to be determined by a posteriori means (given that we are dealing with non-analytic a priori truths). The question that remains is how do we define 'a priori knowledge'; do any logically valid a priori propositions qualify, or only the ones that are true in the actual world? For the time being, let us assume the latter-we will return to the matter later.

Secondly, I hold that a priori reasoning is fallible, but also that a priori knowledge, understood as above, is fallible. By 'a priori reasoning' I mean the rational activity

\footnotetext{
${ }^{1}$ An 'a priori proposition' being any proposition that was reached by a priori means. The validity of the proposition simply means that the reasoning process that led to it is consistent and does not violate the laws of logic, i.e. that human error is ruled out and we have no reasons to suspect its feasibility. 
that human beings engage in when trying to reach a priori knowledge. The Cartesian conception of the a priori maintains a strong link between apriority and necessity, which naturally implies that consistent a priori reasoning provides access to necessary truths. Presumably, this still leaves space for the fallibility of a priori reasoning, but not for the fallibility of a priori knowledge. Kripke's ideas on the matter are usually considered to have severed the link between apriority and modality completely, but these results do not imply that there is no connection between the a priori and modality, they only imply that a priori reasoning is not a direct guide to necessity. There might be a temptation for a deflationary account of the a priori given the usual interpretation of Kripke's results, but we can certainly put forward a more explanatory view if these results are understood correctly. ${ }^{2}$ We have a middle way between abandoning the a priori altogether (cf. Quine 1951, but also MacBride ${ }^{3}$ ) and giving a characterisation of it which does not grasp the traditional sense at all. However, I cannot sympathise with the recent accounts on the nature of the a priori, ${ }^{4}$ for although they do make some important amendments, they tend to fall guilty of the same fault that the classic debate between rationalism and empiricism did, i.e. the illusion that a priori and a posteriori knowledge are wholly separable.

Many modern accounts of the a priori, such as Laurence BonJour's (1998), do correctly acknowledge that a priori reasoning is fallible. There are three things to note here.

1. Human beings are fallible creatures and their rational capabilities are subject to errors.

2. Even when a valid a priori proposition is reached, it might not hold in the actual world.

3. The status of a priori propositions in the actual world is generally determined by a posteriori means, which are, of course, fallible.

The first two points concern the fallibility of a priori reasoning - how it might fail to produce a priori knowledge - whereas the third one suggests, given that a priori knowledge is considered to require the truth of a valid a priori proposition in the actual world, that a priori knowledge as well is fallible. This is a direct consequence of the fallibility of our (empirical) means to verify the truth of any given a priori proposition in the actual world. Accordingly, a priori reasoning can never reach absolute certainty. ${ }^{5}$

What about the supposed empirical indefeasibility of a priori propositions (cf. Field 2000)? Well, in the terms that I have been using, an a priori proposition that is true in the actual world, that is, a logically valid and consistent a priori proposition that counts as a priori knowledge, could still be subject to falsification later. Now, it

\footnotetext{
${ }^{2}$ Friedman's (2000) account, for instance, is a good attempt at this.

${ }^{3}$ MacBride, F., 'Ontological Categories: A priori or A posteriori?', delivered at the Conference On Methodological Issues In Contemporary Metaphysics, 6-7 January 2006, Nottingham.

${ }^{4}$ Cf. BonJour (1998), Peacocke (2000, 2004), Bealer (2000), Field (2000); I will not analyse these accounts in detail, but it will become apparent where my views differ from most recent suggestions.

${ }^{5}$ For the time being, I will leave the case of necessary (non-analytic) a priori truths aside, but they will be discussed briefly later on. However, analytic a priori propositions are not my concern here.
} 
must be noted here that, given a fallibilistic picture to start with, 'truth' is not an absolute notion: the truth of an a priori proposition - unless it is necessary, in which case we will deal with it later - will always be verified by empirical means. Obviously, we might have gotten the empirical story wrong and if this is the case then it would seem that the proposition is not, and never was, true in the actual world. It will still be true in the actual world that the proposition is true in some possible world, just not in this one, but this is another matter. So, it seems that, due to the fallible nature of empirical information itself, there is always a possibility that further empirical information might falsify a priori propositions that were previously believed to be true. This implies that if we insist that 'a priori knowledge' refers to those a priori propositions which are true in the actual world, then a priori knowledge is indeed fallible. I must say that this result does not please me. This is why I will suggest a different definition for 'a priori knowledge'. This definition must be very broad, because otherwise we could never determine when we have reached a priori knowledge. Thus, my suggestion is that any logically valid and consistent a priori proposition constitutes 'a priori knowledge', whether or not it is true in the actual world. When defined like this, a priori knowledge, albeit a very broad notion, is empirically indefeasible and we can avoid the problematic cases where the status of an a priori proposition in the actual world seems to change.

So, a crucial feature of my characterisation of the a priori is the distinction between a priori propositions that hold in the actual world and a priori propositions that grasp merely a non-actual possibility. This distinction is of utmost importance if a plausible characterisation of a priori knowledge is to be established. Without it, we would have no means to deal with cases where an a priori proposition that was believed to be actual is later falsified by further empirical information. The problem is that if we define apriority simply in terms of the actual world, then either the original proposition has lost its a priori status or it was not a priori to start with. Perhaps the best known example of this is the case of Euclidean geometry, which, according to Kant, is a priori and necessary. Empirical results in favour of the general theory of relativity seem to have falsified Euclidean geometry, but it surely cannot be that the a priori status of Euclidean geometry has changed. Either it was always a piece of a priori knowledge and still is or it never was. The consensus seems to be that it was not a priori in the first place, or, at least, Euclid's controversial fifth postulate ${ }^{6}$ is and never was a priori. The problem that we are faced with, however, is that possibly empirical information that falsifies any of Euclid's first four postulates could emerge as well, and again we would have to say that they were not a priori to start with. ${ }^{7}$ This causes two serious complications: a priori knowledge appears to be empirically defeasible, and it seems that we can never know for certain whether we have a genuine piece of a priori knowledge at hand.

A plausible way to deal with these problems is to adopt the distinction between a priori propositions that hold in the actual world and merely possible a priori

\footnotetext{
${ }^{6}$ The fifth postulate states that if two lines intersect a third so that the sum of the interior angles on one side is less than two right angles, then the two straight lines, if extended indefinitely, must intersect on the side on which the sum of the angles is less than two right angles.

${ }^{7}$ Or, perhaps more plausibly, geometry altogether is not a priori, but the point stands. 
propositions (i.e. the ones that do not hold in the actual world), which I suggested above. We ought to keep in mind though that, because of the fallible nature of our verification methods, the status of a priori propositions in regard to the actual world may be subject to revision in the future. Nevertheless, we can agree that once the validity of an a priori proposition is established, that is, if the proposition is logically valid and consistent, its a priori status will never change. So, what happened in the case of Euclidean geometry is that further empirical information pointed out that it does not entirely correspond with the actual world. However, this does not change the a priori status of Euclidean geometry. ${ }^{8}$ So, if my definition of 'a priori knowledge' is accepted, then Euclidean geometry is still very much in the realm of a priori knowledge. This is a small price to pay for a coherent conception of a priori knowledge.

\section{The Bootstrapping Relationship Between A Posteriori and A Priori Knowledge}

Now that the basis of my account has been established, we can advance to examine the first claim: a posteriori and a priori knowledge are in a constant bootstrapping relationship. A dramatic example of this relationship is the gravitational theory. We have good records of how knowledge about gravitation has cumulated in this process. Let us start from Aristotle, who reasoned that the speed of falling bodies is directly proportional to their weight, and thus, heavier bodies should accelerate faster. This appears to be an a priori proposition, but, as Galileo famously argued, two falling bodies of different weights that become connected in the middle of their fall create a paradox in Aristotle's reasoning. This is an example of the fallibility of a priori reasoning due to human error, for no doubt Aristotle believed that he was presenting an a priori proposition. Galileo, however, fared better. Having identified the faults in Aristotle's reasoning, Galileo came up with an a priori proposition of his own, later formulated as a general law for acceleration. Of course, Galileo not only formulated this a priori proposition, but also tried to determine whether it holds in the actual world, as it appeared to do.

For Newton, the level of established a posteriori results was Galileo's theory-a theory which used to be only an unverified a priori proposition, but was verified by a posteriori means. Newton tried to reason how these results could explain the movements of heavenly bodies. His familiar formula states that the gravitational force is proportional to the product of the point masses involved and inversely proportional to the square of the distance between the point masses. This a priori proposition as well seemed to correspond nicely with actuality, as Newton observed in the case of the Moon and the Earth. So, again we have an a posteriori verification for an a priori proposition. Of course, Newton's theory launched numerous attempts to deduce new a priori results about our solar system, and many were successfully established. Among them was the predicted existence of Neptune, which was subsequently found because we knew where to look. However, to get back to the

\footnotetext{
${ }^{8}$ For a more detailed discussion of Euclid's postulates with at least partly similar sentiments, see BonJour (1998, Appendix).
} 
main line of a priori and a posteriori bootstrapping concerning gravitation, we need to advance to Einstein.

Eventually, it became clear that Newton's theory is unable to explain all the movements of the planets. Namely, the orbit of Mercury did not quite seem to follow Newtonian predictions. A posteriori knowledge thus pointed out the insufficiency of an a priori proposition - this is an example of the second sort of fallibility concerning a priori reasoning: Newton's a priori proposition, although valid, did not correspond with the actual world. So we needed a better one, and Einstein gave us general relativity. With the help of general relativity we were able to explain the orbit of Mercury, among quite a few other things. Empirical experiments concerning the bending of light (by sun's gravity) soon corroborated Einstein's theory and its superiority over Newton's theory was obvious. However, we do not need to stop here, for combining general relativity and quantum mechanics has proved out to be very problematic. So, the current situation is that yet again we are looking for an a priori proposition which would explain quantum gravity. There are several suggestions in the air, such as the string theory, but at present we have no means to determine whether the a priori propositions of string theory hold in the actual world or are merely non-actual possibilities.

This is of course a very simplified description of the bootstrapping relationship; for instance, we are not really talking about individual a priori propositions but, rather, about a network of them. All that I am trying to establish here is that knowledge accumulates in a manner of bootstrapping. If you have doubts about whether the reasoning involved in these scientific examples is really a priori, bare with me for a while, as I will elaborate on this. In the meanwhile, I will illustrate the process with another example: a game of chess.

Someone with good knowledge of different openings can play several moves in a chess game with only his experience of these famous openings as a guide. He can simply counter every move by the corresponding move in the opening library, which is based on previous chess games. But when the opponent makes an unexpected move, or when enough moves have been played and the opening library is of no help, even the best chess player has to start thinking about his next move. One must consider different possible move combinations as deep as possible and decide on the best one, analogously to the case of different possible scientific explanations concerning gravitation. Of course, a new 'a posteriori basis' for these considerations is established with each played move, and the cycle starts again. So, each chess move played is an example of the bootstrapping relationship between a posteriori and a priori knowledge. But do not be mislead by this example. Even though a chess game mimics the bootstrapping relationship very nicely, it is clearly not a genuine example - naturally the whole idea of the game must be derived from some mathematical truths which would perhaps qualify as a priori knowledge, but these are not the concern of the player. ${ }^{9}$ Indeed, this is an artificial example and I only use it to illustrate the phenomenon of bootstrapping. I will return to the example briefly in the next section.

\footnotetext{
${ }^{9}$ If we did want a genuine chess-related example of a priori reasoning, we should perhaps look at the invention of chess. However, very little is known of it.
} 
Perhaps the conception of the a priori suggested here does not seem very interesting, for it is beginning to look as if almost all reasoning is a priori reasoning. The upshot of my account is indeed that most scientific and certainly all philosophical reasoning fall in the scope of the a priori, but there are several reasons why this is nevertheless a very interesting understanding of the a priori. Some of these reasons will become apparent in the next section, but we can observe a few already.

Firstly, the manner in which a priori reasoning seems to be tangled with the everchanging a posteriori framework is a crucial insight concerning the scientific method. The process is not simply one of coming up with hypotheses and verifying them, as if the a posteriori framework was a sturdy staircase and every hypothesis is a new step on top of the other. The staircase is not immutable: any previous step might prove out to be rotten. Furthermore, we do not have a single direction that the next step can take - in fact, there are whole parallel staircases with altogether different groundings, and our next step could overlap with any of them. This everchanging staircase is supposed to illustrate the uncertainty concerning the verification of our a priori propositions; the history of science is full of examples. The problem is that we can never know with certainty that the staircase we are building is the actual one, that is, our a priori propositions might have led us astray about what is actual. What is interesting is how we might learn to better evaluate these a priori propositions. This is not necessarily a purely empirical matter, for part of the question is which combinations of different a priori propositions are compatible - it is the task of ontology to examine this. But I am already getting ahead of myself. Before we can discuss these matters in detail, something must be said of another interesting consequence of this conception of the a priori.

The staircase might still serve as a useful metaphor. What is interesting is the first step or, even further, the ground below the staircase. If my understanding of the bootstrapping relationship between a priori and a posteriori knowledge is correct, there is a pressing question about where does all this start. It would appear that the staircase can only be grounded in a priori principles, as each step seems to first require an a priori proposition, which is then checked against experience. Well, there may be some a priori principles, such as the law of non-contradiction, ${ }^{10}$ which could serve as such fundamental principles. If there are principles like this, it would seem that they must be necessary - a common ground for all possible staircases. If this is the case, these principles are obviously of utmost importance for us, as they would tell us something about the necessary constraints of reality, not only about the actual world. But before we get too enthusiastic, a word of caution is in order. It seems that we have no reliable method of testing whether we have indeed reached a fundamental a priori proposition or merely one of the very first steps in our staircase. Clearly, verifying the principle by empirical means only helps in terms of the actual world. This also implies that no matter how irrefutable something like the law of non-contradiction seems, we cannot simply postulate its necessity and infer that it must hold in the actual world as well-even the law of non-contradiction is

${ }^{10} \mathrm{Or}$ at least the law of minimal contradiction, i.e. not every statement is both true and false (cf. Putnam 1978). 
subject to verification, or falsification as the case may be. Having said that, I think that we have a fairly reliable case for the validity of the law of non-contradiction in the actual world, and to my mind it is also our best candidate for a metaphysically necessary principle. Consequently, as groundbreaking as necessary a priori truths would be, it is always quite risky to claim that we have found one.

So, when we engage in a priori reasoning, we take small steps on our staircase towards what could possibly follow from the already established steps. In philosophy, these steps tend to be considerably bigger than in other disciplines (and potentially more erroneous because of that). However, a priori reasoning is what philosophers are educated to do and they, if anyone, should go for the largest steps. The question that we are left with is what grounds these different possibilities that might follow? To answer this and other related questions, we need to examine my second point: the connection between the a priori and modality.

\section{The Modal Basis of A Priori Reasoning}

In the examples of the previous section we saw that a priori reasoning appears to be concerned with possibilities. Consider the chess game again: each of the trillions and trillions of move combinations represents a different possibility, a different path that the game could take. It is by considering different possibilities that we try to determine the path that leads to victory. A chess game, though, is hardly a challenge for our rational capabilities when compared to a priori reasoning concerning the reality. It is a closed system with strict rules and no exceptions. Compared to the number of different possible paths that the reality might take, a chess game seems very simple. In a chess game, our reasoning relies on the rules of the system; how else could we determine the possible routes that the game might take. The question that emerges is: are there analogous 'rules' in reality, that is, constraints for the different possible routes that reality might take?

It would appear that there indeed must be some constraints like this, as otherwise we would be unable to reach any results whatsoever concerning reality. However, even if there are some constraints that restrict the possible organisations of the world, the situation is a lot more complicated than the chess game, not only because the space of possibilities is so much bigger but also because we do not know, exactly, what the constraints are. Recall the distinction between actual and non-actual a priori propositions. I suggested that even if an a priori proposition is logically valid and consistent it still might not hold in the actual world. This would never happen in a chess game. If a move in a chess game is valid, it just means that all the rules of the game have been followed. It might not be a good move, but ontologically its status is identical with all the other valid moves. In contrast to the chess game, an a priori proposition about the reality can easily fail to follow all the constraints because we lack sufficient knowledge about them. The only criterion for the validity of an a priori proposition is that it is logically valid and consistent, i.e. it does not violate the laws of logic and any human errors in the reasoning process are ruled out. Also, as we hope to reach a priori results which are actual and not only possible, the proposition should also be consistent with established a posteriori results. So, we can deem an a priori proposition valid if it was reached by reliable methods-by 
logically valid and consistent reasoning. ${ }^{11}$ If there are no empirical considerations that contradict the proposition, then it is also potentially actual.

As we saw in the story concerning gravitation, Galileo, Newton, and Einstein all put forward propositions which were valid in the sense described above. However, so far, every time it has turned out that the governing conditions of reality are a lot more complicated than we previously believed, as empirical information that conflicts the suggested propositions has emerged. Generally, we could say that science is an attempt to come up with the best approximation that fits these conditions. Metaphysics, on the other hand, examines these conditions. They consist of things like relations between different kinds of entities, identity and existence conditions and other conditions based on the fundamental structure of reality. Of course, the conditions themselves are examined with the help of a priori reasoning. For the most part, such as in scientific contexts, they are presupposed, which is to say that scientists do not contemplate how the conditions work. The identity of Hesperus and Phosphorus was settled by observing the sky, not by contemplating the identity conditions between heavenly bodies. Nevertheless, a set of identity conditions was presupposed.

The role of modality in this process is now starting to emerge. It is the tool that we use to postulate different scenarios of how the governing conditions of reality might work. Consider an example that I mentioned before: the discovery of Neptune. ${ }^{12}$ The discovery of Neptune is usually credited to Le Verrier, a French mathematician who predicted its location from calculations concerning the perturbations in Uranus' orbit. These calculations were of course based on Newton's work on the gravitational force. Quite simply, the perturbations in Uranus' orbit had to be caused by a massive body somewhere nearby. From Newton's formula for the gravitational force, we get the distance between two massive bodies, Uranus and Neptune, so we can roughly determine where Neptune must be. Here, it is Newton's theory of the gravitational force which is the most important part of the process of finding Neptune. His theory of gravitation is a scenario about how the governing conditions of reality might work in regard to massive bodies. It successfully explains some of the factors that affect the relations between two massive bodies; only some, because it turned out that the scenario fails in more general contexts, where the gravitational potential increases. This is where we need to switch to Einstein's scenario.

The modal basis of any given scenario about how the governing conditions of reality might work must be based on the different possible states of affairs that could explain empirical observations. In our example, Le Verrier took advantage of Newton's general theory of how massive bodies interact via gravitation and derived the most plausible case of what could explain the perturbations in Uranus' orbit. This was another massive body, Neptune, situated appropriately. It is important to see that the idea is not just to identify a priori propositions with contingent scenarios concerning the possible states of affairs. The possibility of these scenarios is of a more fundamental sort-just any scenario will not do. According to the account at

\footnotetext{
${ }^{11}$ Possibly also with the help of, say, a computer, as Kripke (1980, 35) has suggested.

12 The example has also been mentioned by Kripke (1980, 79n) and discussed by Hughes (2004, 95-96).
} 
hand, the modality in question is grounded in the governing conditions of reality. In this case, the relevant conditions would concern the relations between massive heavenly bodies: the essences of the entities of this particular kind. This implies that we are working with metaphysical modality. ${ }^{13}$ This is why I have stressed that the modal basis of a priori propositions is so important, for if the modality here would be epistemic or conceptual, it would reduce a priori propositions into statements which have no bearing on the actual governing conditions of reality. This cannot be, as a priori propositions clearly $d o$ have a bearing on these conditions.

A possible objection to this picture can be anticipated. One thing that Kripke has managed to convince most people about is that 'a priori' is an epistemic notion and 'necessity' is a metaphysical notion. Consequently, the connection between a priori knowledge and modality has been deemed to fail. Here I have tried to re-establish that connection in terms of possibility. I also think that Kripke's case only amounts to the conclusion that a priori truths are not always necessary or necessary truths a priori, which I, of course, happily admit. Ultimately, the upshot of my view is that epistemic and metaphysical issues are fundamentally connected, and it is precisely modality that upholds this connection. However, my opponent might insist that the examples concerning scientific hypotheses that we have considered are just that: examples of scientific hypotheses, not of a priori reasoning. Moreover, a devoted empiricist could argue that possibility has little to do with all this; scientific hypotheses are just well-advised guesses, which are then verified or falsified empirically. There is nothing more to the picture, just guesses and empirical research.

However, there is more to the empirical story than meets the eye. Consider an example that I already mentioned in passing: the identity of Hesperus and Phosphorus. I suggested that their identity was settled by observing the sky, by empirical means. I also said that a set of identity conditions was presupposed. What I mean is that it could not have been discovered that Hesperus is Phosphorus if we did not have some criteria of identity for the sortal 'planet'. For example, it must have been known that two planets cannot occupy the same place at the same time. Before we were able to settle whether Hesperus and Phosphorus are actually identical, we had to know that it is possible that they are identical. ${ }^{14}$ Above, I have argued that we need a priori reasoning to determine what is possible. And why cannot a priori reasoning be just guesswork? Well, because what is possible is determined by the identity and existence conditions of the involved entities. The 'guess' has to be based on something, and it can only be based on the natures of the involved entities. Thus, this is not merely to equate a priori propositions with contingent propositions, far from it. Otherwise, a priori reasoning would indeed be indistinguishable from guesswork.

So, before we can settle the actuality of anything, we must already have determined its metaphysical possibility. Metaphysical possibility, I take it, reduces to the essences of the entities concerned. The problem with the empiricist's objection is

\footnotetext{
13 My sympathies are with Kit Fine's (1994, see also Lowe 1998) account of metaphysical modality-it is impossible to go into details here, but as is well known, Fine suggests that we should analyse metaphysical modality in terms of essences rather than the other way around.

14 See Lowe (1998) for an extensive explanation of why possibility precedes actuality. 
that empirical research is committed to this very picture. Without the a priori delimitation of what is possible, we could never reach knowledge about what is actual. A priori reasoning delimits the space of metaphysical possibilities, and only after the initial delimitation has been done can we proceed to test individual a priori propositions by empirical means. This cycle emerges repeatedly, as progress from established empirical results to new information again requires a delimitation of different metaphysically possible states of affairs which are compatible with the current results. Here we have a method by which knowledge slowly but surely accumulates, even though we can never reach absolute certainty.

\section{Conclusion}

I am now in the position to define the a priori with a single phrase: the a priori concerns different metaphysically possible configurations of the governing conditions of reality. Already in Section 1 I suggested that we should define a priori knowledge in the broad sense, that is, all knowledge concerning the different metaphysically possible configurations of the reality is a priori knowledge, even though only one of these configurations is actual. However, given the difficulty of the task of determining which configuration is the actual one, we are better off with a broad definition of a priori knowledge; otherwise, we have very little use for the notion. A priori knowledge in this sense is accessible to all rational human beings, and, as demonstrated in Section 2, it is in a constant bootstrapping relationship with a posteriori knowledge. The aim of metaphysics (and science, I might add) is to establish the actual governing conditions of reality, but this process is fundamentally fallible. Nevertheless, we have good means to falsify a priori propositions which do not hold in the actual world, so we can at least narrow the space of metaphysical possibilities, thus slowly but surely gaining more knowledge about what might be actual, even if the space of metaphysical possibilities does approach infinity.

The exact route from a priori reasoning to knowledge about possible configurations of the governing conditions of reality has not been extensively examined here. It has been shown that metaphysical modality plays an important part in this and it could be said that a priori reasoning just refers to our ability to grasp these metaphysically possible states of affairs, which I take to be grounded in essences. Given this understanding of metaphysical modality, the process is relatively straight-forward: the relations and identity and existence conditions concerning the objects of our inquiry impose constraints to the possible configurations that the reality may take, and the space of possibilities consisting of these configurations is accessible to our a priori capabilities. Modality, then, is what upholds the connection between a priori reasoning and the structure of reality.

The upshot of this characterisation of the a priori is that we can, after all, salvage something of the classic understanding of a priori knowledge. Knowledge acquired with the help of a priori reasoning might not be necessarily true, but it never fails to be possible, insofar as human error is excluded. There may be nothing particularly glorious about a priori knowledge, indeed, as I have argued, much of scientific reasoning falls within its scope, but there is no doubt about its value for philosophy and science, as it is the basis of the scientific method and all philosophical reasoning. 


\section{References}

Bealer, G. (2000) 'A Theory of the A Priori', Pacific Philosophical Quarterly 81: 1-30.

BonJour, L. (1998) In Defense of Pure Reason: A Rationalist Account of A Priori Justification (Cambridge: Cambridge University Press).

Field, H. (2000) 'Apriority as an Evaluative Notion', in Boghossian \& Peacocke 2000: 117-149.

Fine, K. (1994) 'Essence and Modality', J. E. Tomberlin (ed.), Philosophical Perspectives, 8: Logic and Language (Atascadero, CA: Ridgeview), pp. 1-16.

Friedman, M. (2000) 'Transcendental Philosophy and A Priori Knowledge: A Neo-Kantian Perspective, in Boghossian \& Peacocke 2000: 367-383.

Hughes, C. (2004) Kripke: Names, Necessity, and Identity (Oxford: Oxford University Press).

Kripke, S. (1980) Naming and Necessity (Cambridge, Mass.: Harvard University Press).

Lowe, E. J. (1998) The Possibility of Metaphysics (Oxford: Oxford University Press).

Peacocke, C. (2000) 'Explaining the A Priori: The Programme of Moderate Rationalism', in Boghossian \& Peacocke 2000: 255-285.

Peacocke, C. (2004) The Realm of Reason (Oxford: Clarendon Press).

Putnam, H. (1978) 'There Is At Least One A Priori Truth', Erkenntnis 13: 153-70, reprinted in Realism and Reason (1983) (Cambridge: Cambridge University Press).

Quine, W. V. (1951) 'Two Dogmas of Empiricism', The Philosophical Review 60: 20-43, reprinted in From a Logical Point of View (1961), 2nd edn. (Cambridge, Mass: Harvard University Press). 